\title{
Watch Your Step Children! Learning Two-Digit Numbers Through Mirror-Based Observation of Self-Initiated Body Movements
}

\author{
Margina Ruiter $^{1}$ (D) - Sofie Loyens ${ }^{1,2} \cdot$ Fred Paas $^{1,3}$
}

Published online: 25 July 2015

(C) The Author(s) 2015. This article is published with open access at Springerlink.com

\begin{abstract}
It was investigated whether task-related body movements yield beneficial effects on children's learning of two-digit numbers and whether these learning effects are affected by mirror-based self-observation of those movements. Participants were 118 first-graders, who were randomly assigned to two movement conditions and two nonmovement control conditions. In the movement conditions, children were instructed to build two-digit numbers by making and simultaneously verbalizing out loud different sized steps representing the smaller units the numbers consisted of (e.g., the number "36" was construed by saying out loud "10," "20," “30," “35," “36," while making three big steps, one medium, and one small step) on a ruler across the floor. In one of the movement conditions, the children were additionally asked to observe their steps in a mirror. In the first conventionally taught control condition, the children were asked to verbally build and mark the two-digit numbers on a ruler depicted on a sheet of paper. In the second control condition, children were seated before the ruler across the floor, and after verbally constructing the two-digit number, they had to walk to the appropriate position of the number on the ruler across the floor. In the subsequent test phase, children's knowledge of two-digit numbers was assessed by a final math test. The results confirmed the hypothesis that the movement conditions lead to higher test performance than the non-movement condition and revealed that test performance was not differentially affected by mirror-based self-observation.
\end{abstract}

Keywords Embodied cognition $\cdot$ Full-body movements $\cdot$ Self-observation $\cdot$ Learning

Margina Ruiter

ruiter@fsw.eur.nl

1 The Institute of Psychology, Faculty of Social Sciences, Erasmus University Rotterdam, P.O. Box 1738, 3000 DRRotterdam, The Netherlands

2 University College Roosevelt, Middelburg, The Netherlands

3 Early Start Research Institute, University of Wollongong, Wollongong, Australia 
Most of us have been exposed to nursery rhymes sung by parents and teachers when we were young. A well-known example of such a rhyme is "Head, Shoulders, Knees, and Toes." Children are instructed to move while they sing the song, and to touch their head, shoulders, knees, and toes in sequence while singing each word. In this way, they learn to name different body parts. The layman rationale behind those rhymes is (besides the fact that children enjoy those rhymes), that it leads to better learning than simple rehearsal, because it offers a rich sensory experience with tunes, repetition, rhythm, and movement. This study focused on how making body movements and observing one's own movements can facilitate the learning process in young children.

There is a long tradition of considering the role of human motor actions for learning in educational and developmental theories. Piaget was one of the first who emphasized that all actions form the basis of human learning, especially for young children, who initially understand and act upon their environment using mainly sensorimotor actions (Piaget and Inhelder 1969). As a result of the recent rise of theories of embodied cognition, emphasizing the importance of motor information in learning of cognitive tasks has received renewed attention. As discussed by Pouw et al. (2014), embodied theories propose that cognitive processes are grounded in perception and bodily action. In contrast, more cognitivist approaches treat mind and body as separate. Cognitivist approaches assume that cognitive processes are the result of abstract symbol manipulation, that is, the processing of abstract units of information that are not related to, and not grounded in real-world events (Barsalou 1999; Wilson 2002). Embodied theories suggest that higher cognitive processes, like math and language, are ultimately based on sensorimotor codes within a generalized system that was originally developed to control an organism's motor behavior and perceive the world around it (Barsalou 1999). Evidence for this embodied cognition paradigm comes from many different research domains, ranging from research on action semantics (Lindemann et al. 2006), language and reading comprehension (De Koning and Van der Schoot 2013; Zwaan and Taylor 2006), and cognitive neuroscience (Glenberg et al. 2008; Martin 2007). These studies show that visual and motor processes are activated in the brain during the performance of cognitive tasks (e.g., math, reading, problem solving), while semantic codes are active when participants perform motor tasks, suggesting a close interrelationship between cognitive and sensorimotor processes.

The idea that the mind and body are closely interrelated has instigated research to determine how bodily movements can be incorporated in education to improve the effectiveness of learning environments. Recently, Paas and Sweller (2012) have offered an evolutionary account of how body movements can be used to assist the acquisition of knowledge. Using Geary's work (Geary 2002, 2007, 2008) they make a distinction between two types of knowledge: biologically primary knowledge - information we have evolved to acquire over many generations - and biologically secondary knowledge - knowledge that is useful in the social and cultural environment in which a group is situated. Learning to move or face recognition, both very complex tasks in nature, are examples of the first type of knowledge. That sort of learning typifies itself as being unconscious with no need for explicit instruction, effortless, rapid, and driven by intrinsic motivation. Math and reading are examples of biologically secondary information. In contrast to biologically primary knowledge, humans experience great difficulty in learning secondary knowledge. The acquisition of biologically secondary knowledge requires explicit instruction, conscious effort and humans often need to be extrinsically motivated for it. Commonly, the acquisition of this type of knowledge is taught in educational and training contexts. Based on cognitive load theory (Paas et al. 2003a), Paas 
and Sweller (2012) argue that it may be advantageous to use primary information, such as body movements, to assist in the acquisition of secondary information.

\section{Making Movements}

There are different ways to use human movements to foster the learning process. One way of using movements in the educational practice is to instruct participants to "gesture along" during the learning task. Many studies showed that gesturing plays an important role during a mathematical learning task. For example, a study of Cook et al. (2008) revealed that participants who were instructed to make particular gestures while learning a new arithmetical strategy, advanced their learning in comparison with children who were instructed to give verbal explanations during practicing the mathematical problems. Likewise, a study of Broaders et al. (2007) showed that when 9-year-old children were instructed to gesture while they had to solve mathematical problems, they learned more from a lesson by the teacher than when those instructions were not given. These findings suggest that children benefit from gesturing during math instructions. Making gestures while giving verbal explanations may create richer cognitive schemas by adding an additional cue for knowledge representation and retrieval than giving verbal explanations without gestures. Higher-quality cognitive schemas are associated with better learning performance (e.g., van Merriënboer and Sweller 2005). In addition, the construction of higher-quality cognitive schemas from verbal explanations with gestures has been shown to impose less cognitive load than the construction of less complex or lower-quality cognitive schemas from verbal explanations only. Studies in the domain of mathematics (Goldin-Meadow et al. 2001; Ping and Goldin-Meadow 2010) found that making gestures during math problem solving lowers cognitive load, but only when hands are moved in meaningful ways (Cook et al. 2012).

Besides gestures produced by hands, there are some studies that showed that other body movements, such as arm movements, can have a beneficial effect on learning as well. In these studies, written action verbs or phrases were better remembered, when the corresponding actions were performed using any body part compared to when these words and sentences were only re-read or re-heard (Dijkstra and Kaschak 2006; Engelkamp and Jahn 2003; Engelkamp et al. 2004). Thus, when a described action is consciously enacted, its retrieval from memory is facilitated. Another study by Thomas and Lleras (2009) found that arm movements facilitated cognitive performance even if the body movements do not take place during the actual learning task and participants were unaware of the relationship between the body movements and the actual learning task. A study by Shoval (2011) provides another example how body movement can facilitate learning in a cooperation situation with multiple persons. In a geometry class, she investigated "mindful movement" (e.g., children collectively forming a circle with their bodies in order to learn about the circle) and found that the children in the experimental mindful group condition obtained better results than the group taught in a conventional way.

Furthermore, there are two recent studies in which a connection was found between numerical magnitude and motor activity in the form of full-body movements. Using a digital dance mat, Fischer et al. (2011) trained kindergarten children in a number magnitude comparison task to move their whole body in response to a given number. With the task presented on the floor in front of the dance mat, children had to take a step to the right or left to indicate whether the given number was larger or smaller than the standard. Results revealed that the 
spatial-numerical training was more effective than the non-spatial control training in enhancing performance on a number line estimation task. Link et al. (2013) also showed positive training effects after the embodied training in which children were trained to estimate the position of a given number by walking to the appropriate location of that number on a continuous number line on the floor without indications except for the two far ends (i.e., 0 and 100).

Although the making of bodily movements seems to have a beneficial effect on learning, practical applications are still missing, especially in the math curriculum. Given the fact that children nowadays are spending record amounts of time sitting particularly related to school activities (Sturm 2005), which may play an important role in the increase of child obesity (e.g., Butte et al. 2007), it seems crucial to explore possibilities to integrate more full-body movements and not only small body movements (i.e., gesturing) into the learning environment. The present study, therefore, will make an effort to incorporate full-body movements into the standard math curriculum of two-digit number building exercises. Number building exercises are typically given to first-graders as a way to teach numerical concept understanding, such as the positioning of the numbers on a number line, number magnitude, and number structure (see Fig. 1 for an example). The learning of numerical concepts is an important phase in getting first-graders acquainted with mathematics, as it is positively related to their development of more advanced arithmetical skills (Jordan et al. 2007, 2010; Moeller et al. 2011). Integrating full-body movements into these two-digit number building exercises means that children have to build two-digit numbers out of smaller units, with meaningful task-related full-body movements on a number line. The present study will extend the line of research done by Link et al. (2013), which is the only math study that showed that full-body movements are related to enhanced number concept performance. However, the current study deviates from this study by introducing a number line with discrete step size indications (i.e., linear marks of ones, fives, and tens along the entire number line). Doing embodied two-digit number building exercises on this number line introduces the aspect of iterating step by step toward the given decimal number. Furthermore, it provides insight in the way two-digit numbers can be formed from smaller numbers and gives direct visual feedback about the magnitude of these numbers compared to the extent of the number line.

\section{Observing Movements}

Not only making movements by students but also observing movements made by others can aid understanding and facilitate learning. Research conducted by Perry et al. (1995) showed that children learned more about the mathematical concept of equivalence when the teachers' instructions were accompanied by gestures than by verbal instructions only. Similarly, Ping and Goldin-Meadow (2008) revealed that children who had received instruction with speech and guiding gestures (indicating object sizes) in the explanation of a Piagetian conservation task outperformed children who had received instruction with speech only. In the learning of psychomotor tasks, such as paper folding (Wong et al.

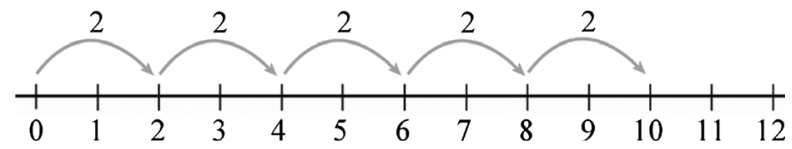

Fig. 1 Example of a number building exercise. Jump to 10 with steps of 2 
2009) or knot tying (Ayres et al. 2009), the positive effects of observing movements have been explained by the existence of a mirror-neuron system (Van Gog et al. 2009). This system, which is assumed to form the basis for the human capability for imitation, is activated by observing motor actions made by others (Rizzolatti and Craighero 2004). Observing someone else perform an action activates the same cortical circuits that are involved in executing an action oneself. Although both action observation and action execution activate the motor system, action observation activates the motor system to a lesser extent than action execution (Keysers and Gazzola 2009). When it is not sensorimotor activation per se, but the strength of the activation that is important, then making movements would be expected to lead to better learning. Indeed, this is idea is supported by several studies (e.g., James and Swain 2011; for studies that do no support this conclusion, see e.g., Marley et al. 2011).

Besides, brain research showed that the same cortical regions involved in motor control are activated when participants have to observe and judge their own videotaped motor actions as when they have to observe and judge other people's videotaped actions (Grezes et al. 2004). The only difference is the onset of this activation. Observing one's own actions in comparison with observing those of others results in earlier activity in action-related areas. This is possibly due to a closer match between the simulated and perceived action (Decety and Grezes 2006).

As both observing and making gestures have been shown to positively affect learning, it would be interesting to explore how the combination of those two elements would contribute to the learning process. One way to combine making and observing movements is to let children initiate movements themselves in front of a mirror and observe their movements in the mirror. In this way, a combination of active moving and observing can be established. Although some studies in the sports and movement domain have examined the role of a mirror in the learning of certain motor skills, to the best of our knowledge, no study has ever looked at the effects of simultaneously making and observing movements in a mirror on learning. However, there is an indication for the positive effect of observing oneself in a mirror for the acquisition of motor skills from a study by Dearborn and Ross (2006). They found that initial dance movements learning in a mirrored setting produced no better performances initially, but better performances in the retest 1 week later. Clearly, the participants found learning the dance movements easier without the mirror, but the information was also more easily lost over the week. In contrast, the mirror learners did not do as well initially, but did improve their performance. According to Dearborn and Ross (2006), these results suggest that a more difficult learning phase induced by a more complex environment increases long-term learning and performance.

Considering these results, it could be argued that the beneficial effects of observational motor learning can be transferred to situations in which self-observation of selfinitiated movements takes place. Furthermore, one could argue that the combination of making and observing movements may lead to an enriched cognitive schema, which not only contains proprioceptive, but also visual content. On the other hand, however, it could also be argued that through the combination of both observing and moving, task complexity is increased to such an extent that it could actually hamper the learning process. In this study, we explored this issue by comparing the full-body movements training with and without mirror-based self-observation on the learning of two-digit number building. 


\section{Present Study}

This study focused on the role of making and observing one's own movements in the learning process of number building skills in children. It was investigated whether making full-body movements either with or without self-observation through a mirror, contributes to two-digit number building learning. We hypothesized that training conditions, in which task-relevant body movements were made, would result in higher learning and test performance than training conditions in which no movements were made (hypothesis 1). In addition, we explored whether mirror-based self-observation of self-initiated body movements and selfinitiated body movements only would differentially affect the learning process. Furthermore, we explored whether the hypothesized beneficial effects of making and observing movements would be mediated by cognitive, affective, or both factors. Therefore, we asked participants to provide a subjective rating on their interest in the training, the difficulty of the training, understanding of the training, and mental effort invested in the training.

\section{Method}

\section{Participants and Design}

Participants were 118 first-grade children $(71$ females, mean age $=7.10$ years, $\mathrm{SD}=0.43$ ) from two Dutch elementary schools. Both schools and their auxiliary branches were situated within a 4-km radius. Children were tested individually outside of the classroom in the gym during the regular school day. This study had four conditions; two movement conditions, i.e., move $(N=28)$ and move and mirror $(N=27)$, and two non-movement, control conditions, i.e., sit $(N=32)$ and write $(N=27)$. All children were randomly assigned over the different conditions and all practiced building twodigit numbers in the 10-50 range with the smaller number units of tens, fives, and ones. After the training phase, all children were tested for their two-digit numbers knowledge with new similar math problems in the 10-99 range on a final math test. For the randomization procedure, the experimenter used the alphabetic naming list the teacher provided, to assign all children from one class to different conditions. Each group of consecutive children on the list was assigned to one of the four conditions. The group size depended on the total number of children the class consisted of. For example, if a class consisted out of 20 children, every condition contained five children.

\section{Materials}

Number Building Exercises-Training In all conditions, children were given the same ten number building exercises on a horizontal number line. The method for doing these number building exercises differed across conditions. In the two movement conditions, first graders were instructed to take steps of different sizes along a horizontal ruler on the floor without numbers, but with smaller, mediate, and larger lines that indicated ones, fives, and tens in order to get them acquainted with two-digit numbers (e.g., 47). ${ }^{1}$ Children were instructed to count while making the steps.

\footnotetext{
${ }^{1}$ Although math curricula vary from school to school, there are guidelines for what a first-grader should learn in math. By the end of first grade, children should be able to add and subtract within 20 and should be able to count at least to 50 by ones, twos, fives, and tens.
} 
Practicing with two-digit numbers on a horizontal line consists of two important meaningful movement-elements: firstly, participants had to make different steps from the left to the right; secondly, the size of the steps are proportionate to the size of the smaller units (ten, fives, and ones) the two-digit numbers consist of. So, tens were represented by large steps, fives by mediate, and ones by very small steps. The math-movement exercise was in line with several experimental studies on mathematical cognition. The first meaningful movement-element of the exercise corresponded with studies showing that numbers are spatially represented (e.g., Fias and Fischer 2005; Hubbard et al. 2005). One prominent theory, the mental number line theory, links numerical magnitudes with positions along the horizontal axis. This theory posits that smaller magnitudes are associated with a position on the left and larger magnitudes on the right of this horizontal line (Restle 1970). Several studies have empirically supported this notion (Dehaene et al. 1993; Fias and Fischer 2005). The second meaningful movementelement of the math-movement exercise was in accordance with empirical findings from different behavioral studies showing inferences between numbers and sized-related information from other cognitive domains like sensorimotor representations of physical size or temporal duration (Walsh 2003). For example, a study of Lindemann et al. (2007) found interferences between number and aperture size while objects were grasped. Precision grip actions were initiated faster in response to relatively small numbers (i.e., 1 and 2), whereas power grips were faster to large numbers (i.e., 8 and 9).

The two non-moving control groups practiced the number building exercises differently. In the first conventionally taught control, children were presented with an A4 sheet on which a ruler was depicted. After calling out loud the smaller units that make up the number, they were instructed to mark the appropriate position of the number on the ruler with a pen. In order to make a good comparison between the move and conventionally taught control condition, as the size of the ruler between conditions differed, this study included a second control condition as well. In this control condition, children had to sit on a chair in front of the ruler and after verbally constructing two-digit numbers, they had to walk to the appropriate position of the number on the ruler across the floor. Note that although in these non-movement control conditions children still have to move (i.e., small hand movement to mark the position on the ruler and walking to the position on the ruler), these movements were not task-related like the movements in the experimental conditions.

Performance on the number building exercises was operationalized by two different scores. The first score (i.e., position score) indicated whether participants indicated the correct/ incorrect position of the two-digit number on the ruler. Children received one point for every correct location. The second score (i.e., instruction score) indicated whether the participants followed the instructions. That is, whether they arrived at the correct answer in as few steps as possible, given the three available number units (i.e., tens, fives, and ones), working from large to small number units. Children received one point for every correctly performed exercise (see Fig. 2 for a graphic representation of the ruler).

Number Building Exercises-Test A final math test was administered to determine the skill level for building two-digit numbers in the 10-99 ${ }^{2}$ range with smaller units of tens, fives, and ones, directly after practicing the number building exercises. The math test comprised two

\footnotetext{
${ }^{2}$ Two-digit numbers $10-50$ were practiced during the learning phase and were therefore considered as neartransfer items on the final math test. Additionally, we examined in this test whether children could extend their understanding to new far-transfer problems in the range 50-99.
} 


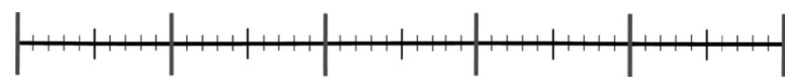

Fig. 2 Graphic representation of the ruler used in this study

parts with five exercises each. In the first part, children had to use different sized monochrome Lego bricks, symbolizing the different numbers units, to build two-digit numbers. For instance, the number " 47 " can be construed with 4 tens (big bricks), 1 five (mediate brick), and 2 ones (small bricks). In the second part, children had to write down the correct two-digit number, corresponding with a row of Lego bricks put down by the experimenter (see Fig. 3 for an example).

Evaluation Questions The children were asked to indicate to what extent they liked the exercises, to what extent they found it difficult, and whether they thought they had understood the exercises correctly. These questions were measured on a five-point Likert scale. In addition, mental effort during the learning and testing phase was measured after each individual item on a five-point Likert scale adapted from Paas (1992) and Paas et al. (2003b), ranging from "very low effort" to "very high effort." This measure gives an indication of the cognitive load children experience and has been used in many educational psychology studies (see Paas et al. 2003a, b; Van Gog and Paas 2008). The mental effort rating scale (RSME) has shown good internal consistency. For instance, Paas et al. (1994) report high reliability coefficients (Cronbach's alpha) of 0.90 and 0.82 for this rating scale in two studies. In addition, the concurrent validity of the nine-point mental effort rating scale appears to be high, as the scale is sensitive enough to detect small variations in task complexity (Van Gerven et al. 2004). In this study, a reliability coefficient (Cronbach's alpha) of 0.71 was found.

\section{Procedure}

The teacher explained to the class that every child would do some math exercises in the gym on an individual basis along with the experimenter. In the gym, the experimenter gave each child a short introduction about constructing two-digit numbers out of smaller units and explained how the different parts of the ruler depicted on an A4-sheet or on the plastic ruler (3.2 $\mathrm{m}$ tall) positioned on the floor were representing 10,5, and 1. Special attention was given to the unit "fives" as not all children were acquainted with this concept. Furthermore, the experimenter emphasized the essential rule; one has to build two-digit numbers by working from bigger units to smaller units in as few steps as possible.

After this standardized introduction, all children performed 12 number building exercises, from which the first two familiarization exercises (i.e., " 18 " and " 41 ") were done together with the experimenter. In the conventionally taught control group, these practice exercises consisted of the experimenter and child verbally building two-digit numbers by saying out loud the smaller units the number consisted of. After having done so, they marked the appropriate position of the number on the ruler depicted on a sheet of paper. In order to avoid children to use their hands, the children were instructed to sit on their hands while verbalizing the different units. In the second control group, the experimenter and child were seated on a chair in front of the ruler across the floor. After the experimenter had presented the number printed on a small card to the child, the first-grader along with the experimenter built two-digit numbers by saying out loud the smaller units the number consisted of. Subsequently, the child walked to the appropriate position of the 
Fig. 3 Example of final math test item " 39 "

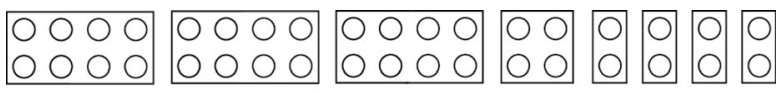

$\left[\begin{array}{l}0000 \\ 0000\end{array}=10\right.$

品 $=5$

定 $=1$

number on the ruler. The no-hands rule applied here as well. In the movement conditions, the experimenter showed how the different sideward steps on the ruler represented 10, 5, and 1, and demonstrated how to move to make up two-digit numbers, while verbalizing the content of the steps. For example the number " 36 " was construed by saying out loud " 10, , " 20 ," " 30 ," "35," "36," while making three big steps, one mediate, and one small step. In the movement condition with mirror (eight connected mirrors of $1.2 \times 0.4 \mathrm{~m}$ ), the experimenter explicitly instructed the children to observe themselves in the mirror while making sideward steps. When this was not the case, the experimenter would stop and ask the child to start the exercise again till he/she eventually managed to simultaneously move and observe

After these two number building practice exercises, every child individually practiced the remaining ten building number exercises as described above. In all conditions, the experimenter stood in front of the gym at the right-hand side (from the perspective of the child) and showed a two-digit number (in the 10-50 range) on a card to the child. During these exercises, the children got no feedback about their performance. After every exercise, the child was asked how much mental effort the exercise required on a five-point Likert scale. The exercises were concluded with a couple of evaluation questions.

Next, the children's knowledge of building numbers was tested on an individual basis by means of a final math test administered in the gym. The children sat behind a table on which three containers filled with different sized monochrome Lego bricks. The biggest Lego bricks of the first container represented tens, the mediate bricks from the second container represented fives, and the smallest Lego bricks out of the last container represented ones. The number units concerned were depicted on the containers. Subsequently, the children were instructed to build two-digit numbers with as few as possible bricks starting with the tens. The bricks had to be placed in a row to resemble the number line exercises.

\section{Data Analysis}

The dependent variables were the math performance scores in both the practice and the test phase. One-way analyses of variance (ANOVA) were performed to determine main effects of the independent variable training condition (move, move and mirror, sit, write), and planned comparisons were performed to determine the specific hypothesized differences between the movement training conditions and the non-movement control conditions. Interest, perceived difficulty, understanding and mental effort were four other dependent variables that were examined with training condition as the between-subjects factor.

\section{Results}

Analyses were completed using SPSS version 21. Alpha was set to 0.05 and Eta-squared $\eta^{2}$ was used as an estimate of effect size, with $\eta^{2}=0.02$ corresponding to a small effect, $\eta^{2}=0.13$ 
corresponding to a moderate effect, and $\eta^{2}=0.26$ corresponding to a large effect (Cohen 1988, 2013). Before conducting analyses, four children who had a score of zero on the final math test, were removed from the dataset. According to information provided by the teachers afterward, these children were all referred to special education due to learning disabilities. Descriptive indices for all variables ordered by condition are shown in Table 1. Mental effort was positively skewed. Math performance in the practice and test phase was negatively skewed. Furthermore, we examined correlations with age and gender. Only gender was related to the final math test scores, $\beta=0.23, t(113)=2.45, p=0.016$, with boys $(M=8.40, \mathrm{SD}=2.39)$, outperforming girls $(M=7.27, \mathrm{SD}=2.73)$. Across all conditions, the number of males varied from 11 to 13 .

\section{Performance During the Different Types of Math Training}

Before testing hypotheses, we compared the performance of the four different types of training during the practice phase. As mentioned before, performance was operationalized by two different scores: an instruction score and a position score. With regard to the instruction score, there was a significant effect of training condition as indicated by the one-way ANOVA, $F(3$, $110)=4.19, p=0.01, \eta^{2}=0.10$. The planned contrast testing the prediction that children who received the math training with movements $(M=7.97, \mathrm{SD}=2.30)$ would outperform children in the non-moving control groups $(M=6.93, \mathrm{SD}=2.40)$ was significant, $t(110)=2.35$, $p<0.001$ (one-tailed), $\eta^{2}=0.04$. A Gabriel post hoc test showed no significant difference in performance between children who received math training with movement and mirror-based self-observation and children who received math training with movement only, $p=0.99$, $\eta^{2}=\sim 0$. Given the descriptive data indicating a possible difference between the two control

Table 1 Mean (SD) descriptive indices

\begin{tabular}{|c|c|c|c|c|}
\hline \multirow[t]{2}{*}{ Measures } & \multicolumn{4}{|l|}{ Training conditions } \\
\hline & Move and mirror $(N=27)$ & Move $(N=28)$ & Write $(N=27)$ & Sit $(N=32)$ \\
\hline \multicolumn{5}{|l|}{ Practice phase } \\
\hline \multicolumn{5}{|l|}{ Performance math training } \\
\hline Position score & $8.93(1.39)$ & $9.14(1.21)$ & $7.11(2.62)$ & $7.97(2.24)$ \\
\hline Instruction score & $8.00(2.27)$ & $7.93(2.32)$ & $6.07(2.54)$ & $7.78(2.25)$ \\
\hline Mental effort & $1.60(0.51)$ & $1.61(0.55)$ & $1.74(0.61)$ & $1.70(0.64)$ \\
\hline Interest & $1.15(0.36)$ & $1.54(1.03)$ & $1.37(0.74)$ & $1.38(0.91)$ \\
\hline Perceived difficulty & $2.00(1.24)$ & $2.00(1.16)$ & $2.04(1.26)$ & $1.94(1.37)$ \\
\hline Understanding & $1.37(0.74)$ & $1.18(0.39)$ & $1.04(0.19)$ & $1.28(0.63)$ \\
\hline \multicolumn{5}{|l|}{ Test phase } \\
\hline Performance final math test & $8.07(2.23)$ & $8.39(2.18)$ & $6.63(2.90)$ & $7.72(2.39)$ \\
\hline Mental effort & $1.48(0.55)$ & $1.64(0.74)$ & $1.61(0.65)$ & $1.51(0.54)$ \\
\hline Interest & $1.12(0.33)$ & $1.21(0.69)$ & $1.22(0.80)$ & $1.16(0.57)$ \\
\hline Perceived difficulty & $1.58(1.03)$ & $1.96(1.32)$ & $1.81(1.30)$ & $1.78(1.13)$ \\
\hline Understanding & $1.19(0.57)$ & $1.14(0.45)$ & $1.22(0.51)$ & $1.34(0.75)$ \\
\hline
\end{tabular}

Mental effort, interest, perceived difficulty, and understanding ratings were measured on a five-point scale. The total maximum score for math performance in both the practice and test phase is 10 
conditions, we also compared the two control conditions with each other on instruction score with a post hoc Gabriel test. The test indicated that the control groups differed significantly at $p=0.04, \eta^{2}=0.13$. A one-way ANOVA for position score revealed a significant effect of training condition, $F(3,110)=6.25, p<0.001, \eta^{2}=0.15$. The planned contrast testing the prediction that children who received the math training with movements $(M=9.04, \mathrm{SD}=$ 1.30) would outperform children in the non-moving control groups $(M=7.54, \mathrm{SD}=2.43)$ was significant, $t(110)=4.09, p<0.001$ (one-tailed), $\eta^{2}=0.13$ (see Table 1). A post hoc Games-Howell test revealed that the difference between the two movement conditions was not significant, $p=0.93, \eta^{2}=\sim 0$. In addition, we performed post hoc comparisons using the Games-Howell test to examine whether the two control conditions differed on position score. The test showed no significant difference between the control conditions, $p=0.34, \eta^{2}=0.03$. To conclude, the movement group outperformed the non-movement control group when building two-digit numbers in the practice phase. Performance during move and mirror training, however, did not significantly differ from performance during the move training.

\section{Performance on Final Math Test}

In this study, we examined whether an embodied training resulted in enhanced performance on a number building task and whether mirror-based self-observation of one's own movements affects performance on that task. We hypothesized that movement conditions would result in higher test performance than the non-movement control conditions. Furthermore, we explored whether the presence of a mirror had an effect on the learning of number building exercises through movements. The $95 \%$ CIs of the move and mirror, move, write, and sit group were $7.21-8.93,7.57-9.21,5.51-7.75$, and 6.88-8.57, respectively.

To compare the effects of the four different types of math training on the final math test performance, a one-way between subjects ANOVA was conducted. There was a statistically significant effect of training condition on final math performance, $F(3,110)=2.70, p=0.05$, $\eta^{2}=0.07$. When comparing the movement conditions $(M=8.23, \mathrm{SD}=2.21)$ with the control conditions $(M=7.18, \mathrm{SD}=2.65)$, the planned contrasts revealed that having math training with movements significantly increased performance on the final math test compared to the nonmovement control groups, $t(110)=2.32, p=0.01$ (one-tailed), $\eta^{2}=0.05$. A post hoc Gabriel test showed no significant difference between the movement conditions with and without selfobservation, $p=0.96, \eta^{2}=\sim 0$. Given the descriptive data indicating a possible difference between the two control conditions, post hoc analyses were conducted. The Gabriel test, however, revealed no significant differences between the two control conditions, $p=0.43, \eta^{2}=$ 0.01 .

Taken together, these results confirmed our hypothesis that children who received math training with movements performed significantly better on the final math test than children who practiced number building exercises without movements. However, it should be noted that the inclusion of a mirror during math training did not affect performance on the final math test.

\section{Interest, Perceived Difficulty, Understanding, and Mental Effort}

To see whether the results above could possibly be explained by affective and cognitive factors such as interest, perceived difficulty, understanding, and mental effort, we performed several one-way between-subject ANOVAs with training condition as the independent variable. We 
found no statistically significant effect of training condition on interest $F(3,110)=1.10, p=$ 0.37 , perceived difficulty $F(3,110)=0.03, p=0.99$, understanding $F(3,110)=1.94, p=0.13$, and mental effort in both the training $F(3,110)=0.38, p=0.77)$ and the testing phase, $F(3$, $110)=0.43, p=0.74$. Hence, there was no difference among the four groups in how much effort they spent to perform the number building task, or in interest, perceived difficulty, and understanding ratings in the training and test phase.

\section{Discussion}

The present study examined whether task-related full-body movements would facilitate the learning process of number building skills (hypothesis 1) and explored whether mirror-based self-observation of self-initiated body movements and self-initiated body movements only would differentially affect the learning process. Hypothesis 1 was confirmed by the results. Children in the movement training conditions outperformed children in the non-movement training conditions during the training phase, and more importantly, on the final math test. These results are in line with previous studies using full-body movements, indicating that these movements can play a facilitating role during mathematical education (Fischer et al. 2011; Link et al. 2013). The study of Link et al. (2013) already revealed the beneficial effects of literally walking the number line, as it allowed for an embodied experience of placing numbers on a continuous number line. In addition to this finding, this study showed that making different sized steps corresponding with the different number units on a discrete number line could also allow for an embodied experience of the architecture of decimal numbers with positive results on the final math test as a result. By this, it joins a vast literature showing a link between numbers and space (Walsh 2003). Furthermore, this study adds to this existing literature, by presenting a new way of integrating full-body movements in the conventional mathematical curriculum of young school-aged children.

In addition, the present study explored whether there was a difference in learning and test performance between the movement conditions with and without mirror-based self-observation. This was not the case. In contrast with the full-body movements, the mirror did not affect performance during the training phase or on the final math test. So, why did this unique combination of observing and self-initiating movements not yield an effect? In this study, we only included an immediate posttest and not a delayed posttest. It is possible that the positive effects of mirror-based learning only become visible on a delayed posttest, as indicated by a study of Dearborn and Ross (2006) in which non-mirror learners outperformed mirror learners initially, while mirror learners surpassed non-mirror learners in performance over time. Future research could use a delayed test to determine the long-term effects of learning math by mirrorbased observation of one's own movements. Another recent study by Post et al. (2013), similar to this study in the aspect of using a combination of observation and making movements (i.e., gesturing) in grammar learning, is consistent with the null results. In this study, children were instructed to simultaneously gesture along with the instruction gestures (i.e., imitation) in the animation. Contrary to their hypothesis, the results showed that children in the gesturing condition performed worse on the posttest than children in the non-gesturing, control condition. A potential explanation for this result was that all children experienced extraneous (ineffective) cognitive load due to the instructions to simultaneously observe and make gestures. A similar explanation could also account for the null findings in this study. Because children had to make and observe their body movements, a so-called dual-task, there 
might have been insufficient working memory capacity left for learning. Numerous dual-task paradigm studies investigating the simultaneous performance of two tasks have showed that our capability to perform tasks in parallel is limited (Kahneman 1973; Wickens 1991). When the demands of the two tasks (e.g., perceptual and motor operations) exceed the individual's processing resources, performance decrements are to be expected, which are often referred to as dual-task costs. Another way in which the mirror condition might have caused extraneous load, is that it is very difficult to mentally transform the mirror-image of the ruler in order to get the correct internal representation and therefore to profit from the combination of making and observing movements.

\section{Active Ingredients of Full-Body Movements}

Although the beneficial role of bodily movements is in line with previous research, we did not find differences among the training conditions in cognitive and affective factors that could explain the observed difference in performance. So, what caused the differences between the moving and non-moving conditions? Zooming in on the full-body movements, one could discern two important active ingredients. Firstly, the conceptually congruent relation between the different sized steps and the different number units could have facilitated number-building learning, which resulted in higher performance on the final math test. This result would be in line with a recent study of Segal et al. (2014) who found that actions that are conceptually congruent with thinking facilitated thinking, and the earlier-mentioned studies showing that moving the body task-specific ways strengthens a systematic association of numbers and space (e.g., Link et al. 2013). Secondly, then, despite functional resemblance, the steps could simply have aided during the number building exercises across the ruler by functioning as a sort of keep-track-of-counting-device. For the reason that in the non-movement conditions children were prohibited to use their fingers as a way to keep track of counting on the ruler, we do not know whether the advantages of making different sized steps in the movement conditions are due to their auxiliary role in counting with the ruler and/or are due to their functional correspondence with different sized number units.

An unexpected finding was the difference in performance between the two control conditions in the training phase. Children in the sit condition performed significantly better during the training phase than children in the write condition. Although this finding might point out a beneficial impact of very minimal movements (walking to the appropriate place in comparison with moving the hand), alternative hypotheses (e.g., children performed better in the sit condition as the different parts on the ruler could be more easily discerned than the smaller parts on the ruler in the write condition) cannot be ruled out. More research is needed to examine this.

\section{Cognitive and Affective Factors}

Another question is why there were no differences among the training conditions in cognitive and affective factors. One possibility is that the rating scales used were not apt for younger children. Rating scale techniques are based on the assumption that people are able to introspect on their affective and cognitive processes and to report the amount of mental effort invested to accommodate the demands imposed by the task. While quite some studies have demonstrated that young and older adults are capable of doing this (e.g., Paas and van Merriënboer 1994), it is possible that younger children might experience difficulties using these introspective selfreport scales. Several studies have observed that younger children tend to respond in an 
extreme manner (i.e., endorsing the options at either end of the response continuum) when asked to use Likert-type rating scales. For example, a study of Chambers and Johnston (2002), which involved children from 5 to 12 years old, showed that younger children when rating subjective states (but not physical states) are more likely to respond that they are either "a lot" or "not at all" happy, whereas older children were more capable of providing graded ratings in the middle of the scale (e.g., "a little" happy).

While this explanation can account for exaggerated extremity preferences for both sides of the response continuum, it does not explain why most children endorsed options at one side. An alternative explanation for the current pattern of results is the social desirability bias; the pervasive tendency to answer questions in a manner that will be viewed favorably by others (Nederhof 1985). Even though no feedback about performance was given during the whole study, it is possible that children wanted to have approval of the experimenter by choosing in their view the most favorable option. Favorable options in this case would be complete understanding, no invested effort, and no perceived difficulty (i.e., the options that make the children look smart). In future research, this social desirability bias should be prevented, for example, by clearly instructing children that high scores on the effort scale are just as "good" as low scores.

Apart from general comments about response styles and introspective abilities of young children, there are other, more specific elements that might have contributed to the current results. First of all, the unexpected high interest ratings among all training conditions and not only among movement conditions can be explained by the fact that the whole study was an interruption of the daily routine. Children were taken out of class individually by an unfamiliar but friendly experimenter and brought to the gym, a place that triggers positive feelings, such as playfulness, in most of the children. In the gym, children were given a novel and interesting task. Given that curiosity often is associated with positive affectivity (e.g., Litman 2005), it could be argued that these extraordinary elements have contributed to the high interest ratings.

Second, despite the lack of research specifically examining concepts such as effort or working load among younger children, we have noted in our research, anecdotally, that firstgraders tend to diffuse the concept mental effort with perceived difficulty. For example, several first-graders mindlessly anticipated the forthcoming mental effort question raised by the experimenter directly after finishing a number building exercise, by saying "very easy" instead of "very low effort" (i.e., "1" on a five-point Likert scale). One could, therefore, assume that the distinction between both concepts is not that obvious for younger children. Future studies would benefit from including a more performance-based measure of mental effort. In performance-based techniques, mental effort is implicitly assessed by presenting children a concurrent secondary task in addition to the primary task. In this procedure, performance on a secondary task is supposed to reflect the level of cognitive load imposed by a primary task. Commonly, the secondary task involves simple activities such as remembering a list of letters or words or activities requiring sustained attention, such as detecting a visual or auditory signal (e.g., Brünken et al. 2003; Goldin-Meadow et al. 2001). Typical performance variables for sustained attention tasks are reaction time, accuracy, and error rate and in the case of a memory task the number of words remembered.

\section{Limitations and Future Studies}

This study has some limitations worthwhile mentioning. First of all, this study did not include a pretest. Therefore, we could not directly assess the level of number building skills first- 
graders had before the training phase and could, as a result, not determine the specific training gains of the different types of training. However, because the participants were randomly assigned to the conditions, we could expect the prior level of number building skills to be equally distributed across conditions. In addition, a well-known important drawback of a pretest, however, is that it possibly confounds the math training, since participants get primed regarding the test items. In order to avoid this priming effect, we opted for a posttest only design.

Furthermore, on basis of the experimental design, we cannot conclude which specific element(s) of the full-body movement exercises facilitated number building skills in the training and test phase. Do full-body movements work through their auxiliary role in counting with the ruler and/or through their functional correspondence with different sized number units? Besides, by forcing children in the control conditions not to use their hands during the number building exercises, they might have been more obstructed in their performance than the experimental groups, as they could not use their hands as a memory and location device. In future studies, it would be interesting to disentangle these separate elements and to use a control group in which children are allowed to use their hands.

Another interesting, related topic for future research would be to examine possible differences between small movements and full-body movements in their effect on number building exercises. For example, does it matter whether the different sized steps are done by feet on a ruler across the floor or are done by a "walking hand" on a printed ruler? Also, within the context of improving health and fitness of young children, it would be interesting to make the mathematical exercise more physically demanding (e.g., increasing the length of the ruler). As numerous studies have found positive effects of (class-room based) physical activity on learning and academic performance (e.g., Donnely and Lambourne 2011), one could argue that task-related movements with an increased intensity would improve mathematical learning even more.

\section{Conclusions and Implications}

This study sought a way to build physical activity into classroom math lessons, not only to increase the amount of physical activity but mainly to make a positive contribution to comprehension of numerical concepts. To conclude, this study showed that the movement training resulted in better number building skills among first-graders. Observing one's own movements in a mirror, however, did not affect performance. Differences in interest, perceived difficulty, understanding, and mental effort cannot account for this effect, because there were no differences between conditions on those variables. Although one must be careful in drawing conclusions with respect to educational practice from a single study, the present findings have important implications for the educational domain. With simply adding full-body movements to conventional math exercises, math performance can be improved in comparison with the predominant "sit and write method." From a practical point of view, these results are also very promising, as full-body movements can be easily integrated into the conventional number building exercises without the need for purchasing costly new teaching methods and materials. Because of this easily applicable and low cost teaching method, teachers might be more likely to integrate the movements into their classrooms. Also, this form of classroom-based physical activity fits the trend towards getting children more physically active in primary schools. 
Open Access This article is distributed under the terms of the Creative Commons Attribution 4.0 International License (http://creativecommons.org/licenses/by/4.0/), which permits unrestricted use, distribution, and reproduction in any medium, provided you give appropriate credit to the original author(s) and the source, provide a link to the Creative Commons license, and indicate if changes were made.

\section{References}

Ayres, P., Marcus, N., Chan, C., \& Qian, N. (2009). Learning hand manipulative tasks: when instructional animations are superior to equivalent static representations. Computers in Human Behavior, 25(2), 348-353. doi:10.1016/j.chb.2008.12.013.

Barsalou, L. W. (1999). Perceptual symbol systems. The Behavioral and Brain Sciences, 22(4), 577-609.

Broaders, S. C., Cook, S. W., Mitchell, Z., \& Goldin-Meadow, S. (2007). Making children gesture brings out implicit knowledge and leads to learning. Journal of Experimental Psychology: General, 136(4), 539-550. doi:10.1037/0096-3445.136.4.539.

Brünken, R., Plass, J. L., \& Leutner, D. (2003). Direct measurement of cognitive load in multimedia learning. Educational Psychologist, 38, 53-61. doi:10.1207/S15326985EP3801_7.

Butte, N. F., Christiansen, E., \& Sorenson, T. I. (2007). Energy imbalance underlying the development of childhood obesity. Obesity, 15, 3056-3066. doi:10.1038/oby.2007.364.

Chambers, C. T., \& Johnston, J. (2002). Developmental differences in children's use of rating scales. Journal of Pediatric Psychology, 27, 27-36. doi:10.1093/jpepsy/27.1.27.

Cohen, J. (1988). Statistical power analysis for the behavioral sciences (2nd ed.). Hillsdale: Erlbaum.

Cohen, J. (2013). Statistical power analysis for the behavioral sciences. Routledge Academic.

Cook, S. W., Mitchell, Z., \& Goldin-Meadow, S. (2008). Gesturing makes learning last. Cognition, 106(2), 1047-1058. doi:10.1016/j.cognition.2007.04.010.

Cook, S. W., Yip, T. K. Y., \& Goldin-Meadow, S. (2012). Gestures, but not meaningless movements, lighten working memory load when explaining math. Language and Cognitive Processes, 27(4), 594-610. doi:10. 1080/01690965.2011.567074.

De Koning, B. B., \& Van der Schoot, M. (2013). Becoming part of the story! Refueling the interest in visualization strategies for reading comprehension. Educational Psychology Review, 25, 261-287. doi:10. 1007/s10648-013-9222-6.

Dearborn, K., \& Ross, R. (2006). Dance learning and the mirror: comparison study of dance phrase learning with and without mirrors. Journal of Dance Education, 6(4), 109-115. doi:10.1080/15290824.2006.10387323.

Decety, J., \& Grezes, J. (2006). The power of simulation: imagining one's own and other's behavior. Brain Research, 1079(1), 4-14. doi:10.1016/j.brainres.2005.12.115.

Dehaene, S., Bossini, S., \& Giraux, P. (1993). The mental representation of parity and number magnitude. Journal of Experimental Psychology: General, 122, 371-396.

Dijkstra, K., \& Kaschak, M. P. (2006). Encoding in verbal, enacted and autobiographical tasks in young and older adults. Quarterly Journal of Experimental Psychology, 59(8), 1338-1345. doi:10.1080/ 17470210600695092.

Donnely, J. E., \& Lambourne, K. (2011). Classroom-based physical activity, cognition, and academic achievement. Preventive Medicine, 52, 36-42. doi:10.1016/j.ypmed.2011.01.021.

Engelkamp, J., \& Jahn, P. (2003). Lexical, conceptual and motor information in memory for action phrases: a multi-system account. Acta Psychologica, 113(2), 147-165. doi:10.1016/S0001-6918(03)00030-1.

Engelkamp, J., Seiler, K. H., \& Zimmer, H. D. (2004). Memory for actions: Item and relational information in categorized lists. Psychological Research, 69, 1-10. doi:10.1007/s00426-003-0160-7.

Fias, W., \& Fischer, M. H. (2005). Spatial representation of numbers. In J. I. D. Campbell (Ed.), Handbook of mathematical cognition (pp. 43-54). New York: Psychology Press.

Fischer, U., Moeller, K., Bientzle, M., Cress, U., \& Nuerk, H. C. (2011). Sensori-motor spatial training of number magnitude representation. Psychonomic Bulletin \& Review, 18, 177-83. doi:10.3758/s13423-010-0031-3.

Geary, D. C. (2002). Principles of evolutionary educational psychology. Learning and Individual Differences, 12 , $317-345$.

Geary, D. C. (2007). Educating the evolved mind: Conceptual foundations for an evolutionary educational psychology. In J. S. Carlson \& J. R. Levin (Eds.), Educating the evolved mind: Conceptual foundations for an evolutionary educational psychology (pp. 1-99). Greenwich: Information Age.

Geary, D. C. (2008). An evolutionarily informed education science. Educational Psychologist, 43, $179-195$.

Glenberg, A. M., Sato, M., Cattaneo, L., Riggio, L., Palumbo, D., \& Buccino, G. (2008). Processing abstract language modulates motor system activity. Quarterly Journal of Experimental Psychology, 61, 905-919. doi:10.1080/17470210701625550. 
Goldin-Meadow, S., Nusbaum, H., Kelly, S. D., \& Wagner, S. (2001). Explaining math: gesturing lightens the load. Psychological Science, 12(6), 516-522. doi:10.1111/1467-9280.00395.

Grezes, J., Frith, C. D., \& Passinghama, R. E. (2004). Inferring false beliefs from the actions of oneself and others: an fMRI study. Neuro-Image, 21, 744-750. doi:10.1016/j.neuroimage.2003.10.014.

Hubbard, E. M., Piazza, M., Pinel, P., \& Dehaene, S. (2005). Interactions between number and space in parietal cortex. Nature Reviews Neuroscience, 6, 435-448. doi:10.1038/nrn1684.

James, K. H., \& Swain, S. N. (2011). Only self-generated actions create sensori-motor systems in the developing brain. Developmental Science, 14(4), 673-687. doi:10.1111/j.1467 7687.2010.01011.x.

Jordan, N. C., Kaplan, D., Locuniak, M. N., \& Ramineni, C. (2007). Predicting first-grade math achievement from developmental number sense trajectories. Learning Disabilities Research and Practice, 22, 36-46. doi: 10.1111/j.1540-5826.2007.00229.x.

Jordan, N. C., Glutting, J., \& Ramineni, C. (2010). The importance of number sense to mathematics achievement in first and third grades. Learning and Individual Differences, 20, 82-88. doi:10.1016/j.lindif.2009.07.004.

Kahneman, D. (1973). Attention and effort. Englewood Cliffs: Prentice-Hall.

Keysers, C., \& Gazzola, V. (2009). Expanding the mirror: vicarious activity for actions, emotions, and sensations. Current Opinion in Neurobiology, 19, 666-671. doi:10.1016/j.conb.2009.10.006.

Lindemann, O., Stenneken, P., Van Schie, H. T., \& Bekkering, H. (2006). Semantic activation in action planning. Journal of Experimental Psychology: Human Perception and Performance, 32, 633-643. doi:10.1037/00961523.32.3.633.

Lindemann, O., Abolafia, J. M., Girardi, G., \& Bekkering, H. (2007). Getting a grip on numbers: numerical magnitude priming in object grasping. Journal of Experimental Psychology: Human Perception and Performance, 33(6), 1400-1409. doi:10.1037/0096-1523.33.6.1400.

Link, T., Moeller, K., Huber, S., Fischer, U., \& Nuerk, H. (2013). Walk the number line - An embodied training of numerical concepts. Trends in Neuroscience and Education, 2(2), 74-84. doi:10.1016/j.tine.2013.06.005.

Litman, J. A. (2005). Curiosity and the pleasures of learning: wanting and liking new information. Cognition and Emotion, 19, 793-814. doi:10.1080/02699930541000101.

Marley, S. C., Szabo, Z., Levin, J. R., \& Glenberg, A. M. (2011). Investigation of an activity-based textprocessing strategy in mixed-age child dyads. The Journal of Experimental Education, 79, 340-360. doi:10. 1080/00220973.2010.483697.

Martin, A. (2007). The representation of object concepts in the brain. Annual Review of Psychology, 58, 25-45. doi:10.1146/annurev.psych.57.102904.190143.

Moeller, K., Pixner, S., Zuber, J., Kaufmann, L., \& Nuerk, H. C. (2011). Early place-value understanding as a precursor for later arithmetic performance - a longitudinal study on numerical development. Research in Developmental Disabilities, 32, 1837-1851. doi:10.1016/j.ridd.2011.03.012.

Nederhof, A. J. (1985). Methods of coping with social desirability bias: a review. European Journal of Social Psychology, 15, 263-280. doi:10.1002/ejsp.2420150303.

Paas, F. (1992). Training strategies for attaining transfer of problem-solving skill in statistics: a cognitive-load approach. Journal of Educational Psychology, 84, 429-434. doi:10.1037/0022-0663.84.4.429.

Paas, F., \& Sweller, J. (2012). An evolutionary upgrade of cognitive load theory: using the human motor system and collaboration to support the learning of complex cognitive tasks. Educational Psychology Review, 24(1), 27-45. doi:10.1007/s10648-011-9179-2.

Paas, F., \& van Merriënboer, J. J. G. (1994). Instructional control of cognitive load in the training of complex cognitive tasks. Educational Psychology Review, 6, 51-71. doi:10.2466/pms.1994.79.1.419.

Paas, F., van Merriënboer, J. J. G., \& Adam, J. J. (1994). Measurement of cognitive-load in instructional research. Perceptual and Motor Skills, 79, 419-430. doi:10.2466/pms.1994.79.1.419.

Paas, F., Renkl, A., \& Sweller, J. (2003a). Cognitive load theory and instructional design: recent developments. Educational Psychologist, 38, 37-41. doi:10.1207/S15326985EP3801.

Paas, F., Tuovinen, J. E., Tabbers, H., \& Van Gerven, P. W. M. (2003b). Cognitive load measurement as a means to advance cognitive load theory. Educational Psychologist, 38, 63-71. doi:10.1207/S15326985EP3801.

Perry, M., Berch, D., \& Singleton, J. L. (1995). Constructing shared understanding: the role of nonverbal input in learning contexts. Journal of Contemporary Legal Issues, 6, 213-236.

Piaget, J., \& Inhelder, B. (1969). The psychology of the child. New York: Basic Books.

Ping, R. M., \& Goldin-Meadow, S. (2008). Hands in the air: using ungrounded iconic gestures to teach children conservation of quantity. Developmental Psychology, 44, 1277-1287. doi:10.1037/0012-1649.44.5.1277.

Ping, R., \& Goldin-Meadow, S. (2010). Gesturing saves cognitive resources when talking about nonpresent objects. Cognitive Science, 34(4), 602-619. doi:10.1111/j.1551-6709.2010.01102.x.

Post, L. S., van Gog, T., Paas, F., \& Zwaan, R. A. (2013). Effects of simultaneously observing and making gestures while studying grammar animations on cognitive load and learning. Computers in Human Behavior, 29(4), 1450-1455. doi:10.1016/j.chb.2013.01.005. 
Pouw, W. T. J. L., Van Gog, T., \& Paas, F. (2014). Educational Psychology Review, 26, 51-72. doi:10.1007/ s10648-014-9255-5.

Restle, F. (1970). Speed of adding and comparing numbers. Journal of Experimental Psychology, 83, $274-278$.

Rizzolatti, G., \& Craighero, L. (2004). The mirror-neuron system. Annual Review of Neuroscience, 27, $169-192$. doi:10.1146/annurev.neuro.27.070203.144230.

Segal, A., Tversky, B., \& Black, J. (2014). Conceptually congruent actions can promote thought. Journal of Applied Research in Memory and Cognition, 3, 124-130. doi:10.1016/j.jarmac.2014.06.004.

Shoval, E. (2011). Using mindful movement in cooperative learning while learning about angles. Instructional Science, 39(4), 453-466. doi:10.1007/s11251-010-9137-2.

Sturm, R. (2005). Childhood obesity - what we can learn from existing data on societal trends, part 1. Preventing Chronic Disease, 2, 1-9.

Thomas, L. E., \& Lleras, A. (2009). Swinging into thought: directed movement guides insight in problem solving. Psychonomic Bulletin \& Review, 16, 719-723. doi:10.3758/PBR.16.4.719.

Van Gerven, P. W. M., Paas, F., Van Merriënboer, J. J. G., \& Schmidt, H. G. (2004). Memory load and the cognitive pupillary response in aging. Psychophysiology, 41, 167-174. doi:10.1111/j.1469-8986.2003. 00148.x.

Van Gog, T., \& Paas, F. (2008). Instructional efficiency: revisiting the original construct in educational research. Educational Psychologist, 43, 16-26. doi:10.1080/00461520701756248.

Van Gog, T., Paas, F., Marcus, N., Ayres, P., \& Sweller, J. (2009). The mirror neuron system and observational learning: implications for the effectiveness of dynamic visualizations. Educational Psychology Review, 21, 21-30. doi:10.1007/s10648-008-9094-3.

Van Merriënboer, J. J. G., \& Sweller, J. (2005). Cognitive load theory and complex learning: recent developments and future directions. Educational Psychology Review, 17, 147-177. doi:10.1007/s10648-005-3951-0.

Walsh, V. (2003). A theory of magnitude: common cortical metrics of time, space and quantity. Trends in Cognitive Sciences, 7(11), 483-488. doi:10.1016/j.tics.2003.09.002.

Wickens, C. D. (1991). Processing resources and attention. In D. L. Damos (Ed.), Multiple-task performance (pp. 3-34). Bristol, PA: Taylor \& Francis.

Wilson, M. (2002). Six views of embodied cognition. Psychonomic Bulletin \& Review, 9, 625-636. doi:10.3758/ BF03196322.

Wong, A., Marcus, N., Ayres, P., Smith, L., Cooper, G. A., Paas, F., \& Sweller, J. (2009). Instructional animations can be superior to statics when learning human motor skills. Computers in Human Behavior, 25(2), 339347. doi:10.1016/j.chb.2008.12.012.

Zwaan, R. A., \& Taylor, L. J. (2006). Seeing, acting, understanding: Motor resonance in language comprehension. Journal of Experimental Psychology: General, 135(1), 1-11. doi:10.1037/0096-3445.135.1.1. 